\title{
Review of: "Model Study of a Single Pile With Varied Configuration in Sand"
}

\author{
Bilal Malik
}

Potential competing interests: The author(s) declared that no potential competing interests exist.

The scopes and objectives of the study need to be properly identified and elaborated.

The conclusions presented in the study can be found from many previous studies carried out on this topic. The technical contribution of the manuscript is very limited. The authors should clearly indicate what the significant outcome of the study is. 